\title{
Early clinical experience with the new Amplatzer Ductal Occluder II for closure of the Persistent Arterial Duct (PDA)
}

\author{
Goonetilleke MPB, Jayaratne AU, Inthisar HAM, Vithanage ND, Wimalagunarathne KWR, Janzs \\ PU, Pathinayake AD, Anuruddha KBC \\ Paediatric Cardiology Unit, Teaching Hospital Karapitiya, Galle, Sri Lanka.
}

Correspondence: Dr. Mangalanath Goonetilleke

e-mail: mangalagoonetilleke@yahoo.com

\begin{abstract}
Introduction: Transcatheter PDA closure has gained acceptance over surgery because of its cosmetic benefits, shorter hospital stay and absence of pain associated with a thoracotomy. The Amplatzer Ductal Occluder II (ADO II) device provides a solution for the closure of small to moderate-sized PDAs
\end{abstract}

Objective: To describe early single-centre clinical experience with ADO II.

Methods: Children with a haemodynamically significant patent ductus arteriosus (PDA) who underwent percutaneous trans-pulmonary closure with Amplatzer Ductal Occluder II (ADO II) were included. Data was collected from computer based patient records.

Results: Trans-pulmonary PDA closures using ADO II were undertaken in 65 children ( 46 females) with a mean age of 2 years 3 months (range 5 months to 14 years) and a mean weight of $9.6 \mathrm{~kg}$ (range $4.2-25 \mathrm{~kg}$ ).

Complete occlusion was noted pre-discharge in 64 (98.4\%) patients. One (1.6\%) had residual shunting after deployment followed by embolization to the left pulmonary artery on the third day of the procedure. Of the 65 children, five (7.7\%) had mild flow acceleration in the left pulmonary artery and another one (1.6\%) had mild aortic flow obstruction following the procedure.

At 7 and 30 days, echocardiography confirmed complete ductal occlusion without need for further intervention in all $64(98.4 \%)$ successful cases.

Conclusions: ADO II is highly effective in rapid occlusion of morphologically varied small to moderate-sized PDAs.

Key words: Ductal occluder; patent ductus arteriosus; trans-pulmonary; residual shunting)

\section{Introduction}

The reported incidence of patent ductus arteriosus (PDA) in infants ranges from 0.138 to 0.8 per 1000 live births $(1,2)$. Transcatheter PDA closure has gained acceptance over surgery because of its cosmetic benefits, shorter hospital stay and absence of pain associated with a thoracotomy. Since the initial description by Portsmann et al of nonsurgical closure of PDA the percutaneous approach has become standard clinical practice (1). Coils and devices have broadened the range of patients and anatomies treatable with percutaneous techniques (3-6). The ADOII device provides a solution for the closure of small to moderate-sized PDAs. The ADOII device is a modification of the ADOI device as produced by AGA Medical Corporation. It is one of the ranges of vascular occlusion devices based on nitinol wire meshes shaped in sequential lobes. 
ADOII is characterized by two low-profile retention discs for placement in the aorta and pulmonary artery (PA). Connecting waist of variable diameter and length for positioning within the PDA itself is attached to the retaining discs by two articulations. This allows the positioned, relatively soft device to adapt to the patient's anatomy rather than distorting the anatomy to its shape. The aim of the study was to describe the early single-centre clinical experience with the Amplatzer Ductal Occluder II (ADO II)

\section{Methods}

Children presenting with a haemodynamically significant PDA to Teaching Hospital Karapitiya (THK), Galle, Sri Lanka, who underwent percutaneous closure with the ADOII were included in this study. In instances where the PDA was larger than $5.5 \mathrm{~mm}$ in diameter, use of the device was not considered. Patients with additional cardiac anomalies requiring surgical correction were referred for surgery as were patients $<3 \mathrm{~kg}$ in weight. Data collected from computer based patients' records included demographic, clinical, and echocardiographic parameters.

The trans-pulmonary technique for device deployment was adopted. Initially an aortogram was performed using a pigtail catheter. The sizing of the device is based upon the waist diameter and length measured by angiography (Figure 1). The duct is crossed from the PA and the aortic retainer is then exposed in the main aorta and the delivery system withdrawn to allow for the waist to be deployed in the duct itself. The pulmonary retainer is then exposed against the pulmonary arterial wall. Device positioning and residual PDA leaks were confirmed by aortic angiogram (Figure 2). All patients were treated with pre-procedural intravenous cefuroxime (30 $\mathrm{mg} / \mathrm{kg}$ ) with additional three doses postprocedurally. Intravenous heparin $(50 \mathrm{U} / \mathrm{kg})$ was administered at the commencement of each procedure. All patients underwent echocardiography on the next day, after 7 days and at 30 day follow-up visits.

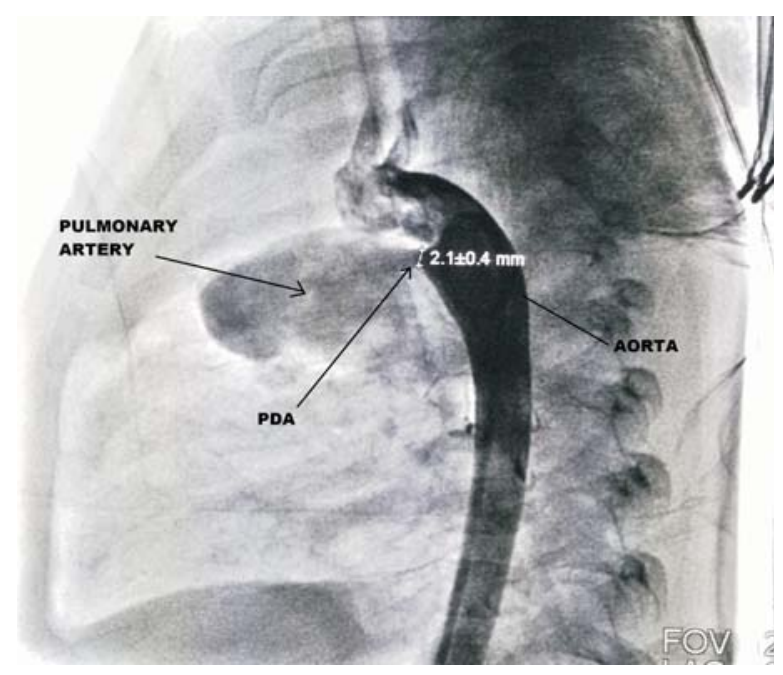

Figure 1: Angiogram to visualize the PDA

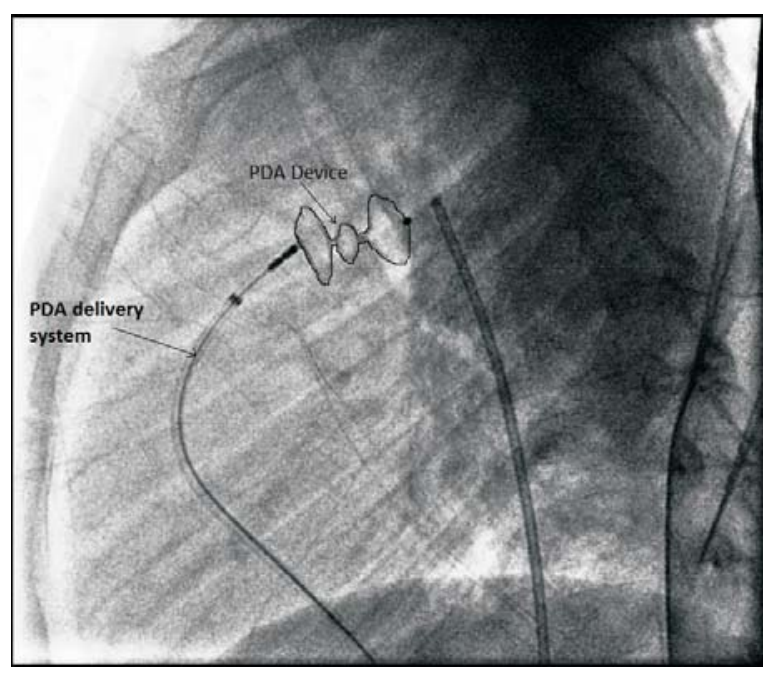

Figure 2: PDA device after deployment

\section{Results}

The frequency of age group distribution is given in Table 1.

Table 1: Frequency of age group distribution

\begin{tabular}{lc}
\hline Age group & No. of patients \\
\hline$\leq 1$ year & 26 \\
1-2 years & 17 \\
2-5 years & 17 \\
5-10 years & 04 \\
Above 10 & 01 \\
\hline
\end{tabular}


Of the 65 patients, 46 were females. Fifteen patients had weights from $3.1-6 \mathrm{~kg}, 31$ had weights from 6.1 $-10 \mathrm{~kg}, 13 \mathrm{had}$ weights from $10.1-15 \mathrm{~kg}, 3 \mathrm{had}$ weights from $15.1-20 \mathrm{~kg}$ and 3 patients had weights above $20 \mathrm{~kg}$.

Angiographic measurement of the PDA size and the PDA device sizes are shown in Table 2 and 3 respectively.

Table 2: Angiographic measurement of the PDA size

\begin{tabular}{cc}
\hline PDA size (mm) & No. of patients \\
\hline $1-2$ & 26 \\
$2.1-3$ & 24 \\
$3.1-4$ & 11 \\
$4.1-5$ & 3 \\
$>5.1$ & 1 \\
\hline
\end{tabular}

Table 3: PDA device size

\begin{tabular}{cc}
\hline Device size (mm) & No. of patients \\
\hline $6 \times 6$ & 16 \\
$6 \times 5$ & 01 \\
$6 \times 4$ & 17 \\
$5 \times 6$ & 14 \\
$5 \times 4$ & 08 \\
$4 \times 6$ & 02 \\
$4 \times 4$ & 04 \\
$3 \times 6$ & 03 \\
\hline
\end{tabular}

From May 2012 to January 2016 trans-venous PDA closures using ADOII were undertaken in 65 children. $6 \mathrm{mmX} 4 \mathrm{~mm}$ ADOII was the commonly used device in our series. Mean age was 2 years and 3 months (range 5 months to 14 years) with a mean weight of $9.6 \mathrm{~kg}$ (range $4.2-25 \mathrm{~kg}$ ). The mean ductal diameter was $2.55 \mathrm{~mm}$ (range 1.1-5.1mm). ADO II was released in all 65 children.
One $(1.6 \%)$ patient had residual shunting after deployment of ADO II followed by embolization to the left PA on the third day of the procedure. This child underwent surgical closure of the PDA after the surgical removal of the embolized device. Complete occlusion was noted pre-discharge in the remaining $64(98.4 \%)$ children. Five of these 65 children had mild flow acceleration in the left pulmonary artery (LPA) and another had mild aortic flow obstruction following the procedure. These 6 children are doing well at present with good weight gain despite still having mild LPA origin narrowing and mild obstruction to the descending aortic flow due to the presence of the device. These are common complications following PDA device closure and are usually overcome with growth of the pulmonary artery. At 7 and 30 days, echocardiography confirmed complete ductal occlusion without need for further intervention in all 64(98.4\%) successful cases.

\section{Discussion}

Transcatheter closure procedures have been performed with high success rates both in children and adults (3-6,8-18). Due to advances in diagnostic and therapeutic modalities diagnosis and treatment of PDA can be accomplished at a relatively early age (5-9). Thanopoulos et al (15) reported success rates of $95 \%$ and $98 \%$ soon after and one month after device implantation in patients with a mean age of 3.6 years.

The ADOII device provides a solution for the closure of small to moderate-sized PDAs in children $(14,15)$. We have demonstrated that percutaneous treatment with ADOII can be realized with lower complication and residual shunt rates. The immediate and short term follow up success rates (complete occlusion) for duct occlusion by ADOII at 24 hours, 7 days and 1 month were $98.4 \%$ all in our series which compares favourably with data reported using Amplazter ADOII devices (8). The multiple layers of nitinol allow for rapid ductal occlusion which reduces residual shunt risks. The ability to position the ADOII such that each of the retention discs assumes its independent orientation significantly reduces the risk of protrusion, anatomical distortion and displacement which has resulted in a $98.4 \%(n=65)$ success rate of device deployment in our series with only one $(1.6 \%)$ re-intervention due to device 
dislodgement which compares favourably with reported data (8).

The ADOII device was designed by the manufacturers with the express intention that the retaining discs would be positioned in the aorta and PA. However, in the presence of a large ampulla, the aortic retaining disc will be positioned within the ampulla and not the aorta. This position makes the aortic disc to appear spherical while the pulmonary disc to be flattened giving a disfigured orientation after deployment although this is the accepted configuration in case of a PDA with a large ampulla. When approached transvenously which we practice coupled with the soft and flexible nature of the Amplazter ADO II devices, the aortic disc can be well positioned in the ampulla without protruding to the aorta, while still allowing the rest of the device to assume its intended position. This has resulted only $1.6 \%(n=1)$ of cases with mild obstruction to the aortic flow in our series.

In case of a short duct, the central waist may protrude into the PA, leaving the PA retaining disc redundant. This occurs even more so if the device is significantly oversized. As such, the shorter device will usually be applicable unless the duct is extremely long. In our experience, this positioning was problematic only in five cases $(7.7 \%)$ with pressure gradients ranging from $10-22 \mathrm{mmHg}$ noted across the left PA origin. Correct and high quality ductal imaging techniques available in our unit allowed us to accurately assess the ductal anatomy and the size which helped us to select the appropriate sized device thus preventing any unwanted pulmonary flow obstructions.

Embolization during release of the device is one of the important complications of the procedure (7-9). Embolization can occur in systemic and generally in pulmonary artery (7-9). In a published series of 27 cases, Forsey et al. (8) reported displacement of the device $(n=1)$ and embolization $(n=1)$. They attributed development of these complications to underestimation of ductal diameter secondary to spasm of the ductus arteriosus induced by catheterization. In our study, as a procedural complication only one (1.6\%) device embolization to the PA was observed three days after the device deployment.Although the selected size of the device matched with the measurements of the acquired ductal images we assume this to be attributable to underestimation of ductal diameter secondary to spasm of the ductus arteriosus induced by catheterization.

Vascular injury to the femoral artery and the vein at the local access site, haematoma formation and damage to the local soft tissues have been reported in published data (5-8). In our series all cases had palpable popliteal pulses 6 hours following the procedure and intravenous heparin infusion was not required in any child.Infection of the local vascular access site was not reported and vascular surgical intervention was not needed in our series. Although this is mainly attributable to the operator experience the ADOII design and flexibility has also allowed delivery with 4 or $5 \mathrm{~F}$ delivery catheters with minimal local tissue and vascular injury as compared to ADO I which needs a minimum of $6 \mathrm{~F}$ delivery system to deliver the smallest available device.

\section{Conclusions and recommendations}

- ADO II is highly effective at providing rapid occlusion of morphologically varied small to moderate-sized PDAs.

- Occluder design allows delivery with 4 or $5 \mathrm{~F}$ delivery catheters with minimal local tissue and vascular injury.

- Stable occluder position is dependent on accurate device sizing correct positioning of both aortic and pulmonary discs, good quality imaging to visualize device configuration and operator experience.

- Flexibility of the articulations allows this device to simplify the treatment in a range of patients and specific ductal anatomies that are more challenging.

- A larger range of sizes and configurations of this occluder may be required to successfully occlude all ductal sizes and morphologies.

\section{References}

1. Fyler DC, Buckley LP, Hellenbrand WE, et al. Report of the New England Regional Infant Cardiac Program. Pediatrics 1980; 65(Suppl):398.

2. Botto LD, Correa A, Erickson JD. Racial and temporal variation in the prevalence of heart defects. Pediatrics 2001; 107(3):1. 
3. Cambier PA, Kirby $W C$, Wortham DC. Moore JW. Percutaneous closure of the small (less than $2.5 \mathrm{~mm}$ ) patent ductus arteriosus using coil embolization. American Journal of Cardiology 1992:69:815-6.

4. Rosenthal E, Qureshi SA, Reidy J, et al. Evolving use of embolisation coils for occlusion of the arterial duct. Heart 1996:76:525-30.

5. Pass RH, Hijazi Z, Hsu DT, et al. Multicenter USA Amplatzer patent ductus arteriosus occlusion device trial: Initial and one-year results. Journal of the American College of Cardiology 2004:44:513-9.

6. Gudausky TM, Hirsch R, Khoury PR, Beekman RH. Comparison of two transcatheter device strategies for occlusion of the patent ductus arteriosus. Catheterization and Cardiovascular Interventions 2008:72:675-80.

7. Pass RH, Hijazi Z, Hsu DT, Lewis V, Hellenbrand WE. Multicenter USA Amplatzer patent ductus arteriosus occlusion device trial: initial and one-year results. Journal of the American College of Cardiology 2004;44:513-9.

8. Forsey J, Kenny D, Morgan G, Hayes A, Turner M, Tometzki A, et al. Early clinical experience with the new Amplatzer Ductal Occluder II for closure of the persistent arterial duct. Catheterization and Cardiovascular Interventions 2009;74:615-23.

9. Bilkis AA, Alwi M, Hasri S, Haifa AL, Geetha K, Rehman MA, et al. The Amplatzer duct occluder: experience in 209 patients. Journal of the American College of Cardiology 2001; 37:258-61.

10. Santoro G, Gaio G, Carrozza M, Palladino MT, Russo MG, Calabrò R. Large patent ductus arteriosus closure with multiple controlled-release coils. International Journal of Cardiology 2007;116:425-6.
11. Spies C, Ujivari F, Schräder R. Transcatheter closure of a 22 $\mathrm{mm}$ patent ductus arteriosus with an Amplatzer atrial septal occluder. Catheterization and Cardiovascular Interventions 2005; 64:352-5.

12. Prada F, Mortera C, Bartrons J, Rissech M, Jiménez L, Carretero J, et al. Percutaneous treatment of atrial septal defects, muscular ventricular septal defects and patent ductus arteriosus in infants under one year of age. Rev Esp Cardiol 2009; 62:1050-4.

13. Park YA, Kim NK, Park SJ, Yun BS, Choi JY, Sul JH. Clinical outcome of transcatheter closure of patent ductus arteriosus in small children weighing $10 \mathrm{~kg}$ or less. Korean Journal of Pediatrics 2010;53:1012-7.

14. Baspinar O, Irdem A, Sivasli E, Sahin DA, Kilinc M. Comparison of the efficacy of different-sized Amplatzer duct occluders (I, II, and II AS) in children weighing less than 10 kg. Pediatric Cardiology 2013;34:88-94.

15. Thanopoulos BV, Eleftherakis N, Tzannos K, Stefanadis C, Giannopoulos A. Further experience with catheter closure of patent ductus arteriosus using the new Amplatzer duct occluder in children. American Journal of Cardiology 2010;105:1005-9.

16. Porstmann W, Wierny L, Warnke H. Closure of persistent ductus arteriosus without thoracotomy. Ger Med Mon 1967;12:259-61.

17. Jacob JLB, Braile DM. Current treatment of the persistent arterial duct. Rev Bras Cir Cardiovasc 2003;18:350-8.

18. Pass RH, Hijazi Z, Hsu DT, Lewis V, Hellenbrand WE. Multicenter USA Amplatzer patent ductus arteriosus occlusion device trial: initial and one-year results. Journal of the American College of Cardiology 2004; 44:513-9. 